\title{
Deoxynivalenol and Nivalenol Accumulation in Wheat Infected with Fusarium graminearum During Grain Development
}

\author{
Megumi Yoshida and Takashi Nakajima
}

Research Team for Fusarium Head Blight Control, National Agricultural Research Center for Kyushu Okinawa Region (KONARC), 2421

Suya, Koshi, Kumamoto 861-1192, Japan.

Accepted for publication 5 April 2010.

\section{ABSTRACT}

Yoshida, M., and Nakajima, T. 2010. Deoxynivalenol and nivalenol accumulation in wheat infected with Fusarium graminearum during grain development. Phytopathology 100:763-773.

The manner in which deoxynivalenol (DON) and nivalenol (NIV) accumulation progresses in wheat grain infected with Fusarium graminearum and the influence of the time of infection on the accumulation of toxins were investigated. Four cultivars were tested in a greenhouse environment, where the plants were spray inoculated at three different stages with a mixture of DON and NIV chemotypes of $F$. graminearum. The results indicate that high levels of DON and NIV can be produced beyond 20 days after anthesis (DAA), even by early infection. The results of field experiments performed on seven cultivars, where inoculation was conducted using colonized maize kernel inoculum, were consistent with the greenhouse results. In addition, in the greenhouse experiments, late infection, at least as late as 20 DAA, caused grain contamination with these toxins even without clear disease symptoms on the spike. These results indicate the importance of the late stage in grain development in DON and NIV contamination, suggesting that control strategies that cover the late as well as the early stage of grain development should be established to effectively reduce the risk of these toxins' contaminating wheat.

Additional keywords: Fusarium head blight, Gibberella zeae, mycotoxin, trichothecene, Triticum aestivum.
Fusarium head blight (FHB) or scab, caused by several Fusarium spp., especially Fusarium graminearum Schwabe (teleomorph Gibberella zeae (Schwein.) Petch), is a widespread and destructive disease of wheat (Triticum aestivum L.), barley, and other small-grain cereals $(24,33,35)$. These pathogens infect spikes and reduce grain yield and quality. Moreover, Fusarium spp. that cause FHB produce trichothecene mycotoxins such as deoxynivalenol (DON) and nivalenol (NIV), which are toxic to humans and other animals.

Trichothecene metabolite profiles (chemotypes) for $F$. graminearum are strain specific; some strains produce mainly DON, whereas others produce mainly NIV $(17,22,41,51)$. DON is the most prevalent mycotoxin in cereals $(1,36)$, and DON chemotypes of Fusarium prevail worldwide. NIV chemotypes are more restricted $(19,29)$; therefore, more attention is generally focused on DON than on NIV as a trichothecene mycotoxin. The Joint WHO/FAO Expert Committee on Food Additives evaluated the risk of DON and set a provisional maximum tolerable daily intake of DON as $1 \mu \mathrm{g} / \mathrm{kg}$ of body weight in 2001 (1). Accordingly, the Japanese government determined provisional guidelines for DON content in unpolished wheat grains as $1.1 \mathrm{mg} \mathrm{kg}^{-1}$ in 2002. Many other countries have also established maximum allowed levels for DON in cereals and cereal products (6).

Although there are not yet guidelines or regulations for NIV, it is also an important Fusarium mycotoxin that frequently occurs worldwide $(36,48)$ and commonly co-occurs with DON $(36,48$, $49,55,56)$. In Japan, where $F$. graminearum is the main causal organism of FHB (20), both chemotypes of $F$. graminearum prevail $(16,31,44,55,56)$, and natural contamination of wheat with

Corresponding author: T. Nakajima; E-mail address: ntakashi@affrc.go.jp

doi:10.1094/PHYTO-100-8-0763

(c) 2010 The American Phytopathological Society both DON and NIV commonly occurs $(48,49,55,56)$. More importantly, NIV is reported to be more toxic to animals than is DON $(39,47,50)$. Therefore, control measures that are effective for both DON and NIV should be established.

Mycotoxin accumulation in grain is influenced by various factors such as the strain of the pathogen, host resistance, duration of head wetness, and other environmental factors $(9,10,15,21$, $23,25,27,28,43)$. Although chemical control, cultural control, and use of cultivar resistance are effective for control of FHB and the accompanying toxin contamination, no single measure has been fully or consistently effective (35). To develop highly effective strategies for controlling FHB, particularly for reducing the risk of toxin contamination, it is necessary to better understand the manner in which trichothecene toxin accumulation occurs in wheat grain. In particular, it is important to know when fungal toxin production mainly occurs during grain development because if we can control toxin production during this period, the final toxin level would be effectively reduced. Information on the influence of infection timing on toxin accumulation is also important because wheat is most susceptible to FHB during anthesis $(3,33,37,46)$ or from anthesis to the early milk stage $(13,32)$ but Fusarium infection can occur at any time up to the dough stage $(3,11,15,21,46)$.

Several studies have investigated the mode of progression of trichothecene mycotoxins in wheat grain, especially for DON, during grain development $(2,7,26,40)$. However, most are based on single-year experiments, and the results have not been consistent. Further research on this subject, including NIV as well as DON accumulation, is required. Although a few studies $(11,15,21)$ have examined the influence of infection timing on levels of DON accumulation, no reports have addressed the influence of infection timing on NIV accumulation in grain.

The objectives of the present study were to (i) investigate the manner in which progression of DON and NIV accumulation occurs in wheat grain infected with $F$. graminearum and (ii) 
examine the influence of infection timing on accumulation of these toxins in grain as well as disease severity.

\section{MATERIALS AND METHODS}

Greenhouse experiments. Four wheat cultivars with different resistance levels to FHB were tested in a greenhouse environment in Koshi $\left(32.9^{\circ} \mathrm{N}, 130.7^{\circ} \mathrm{E}\right)$, Kumamoto, Japan, in spring 2004 and 2007. The cultivars were 'Sumai 3' (resistant), 'Nobeokabouzu' (resistant), 'Emblem' (susceptible), and 'Norin 61' (intermediate).

The two resistant cultivars are widely used as sources of FHB resistance $(38,42)$. Norin 61 is one of the most widely grown Japanese cultivars used to make Japanese noodles. The resistance levels and pedigree of these cultivars are listed in a previous study (5). The pedigree and origin are quite different among these cultivars. All the cultivars are spring wheat genotypes, although Norin 61 is cultivated as winter wheat in Japan. Sumai 3, Nobeokabouzu, Norin 61, and Emblem are soft red, hard red, soft red, and hard white cultivars, respectively.

The cultivars were planted in $18-\mathrm{cm}$-diameter plastic pots $(5$ to 6 plants/pot) at the beginning of December 2003 and 2006 and grown in a greenhouse under natural light until inoculation (April to May in 2004 and 2007). The greenhouse was roofed with a polyolefin film (PO muteki; MKV Platec, Ltd., Tokyo). Average temperatures in the greenhouse from seeding date $(2$ December 2003 and 1 December 2006) to first inoculation date (14 April 2004 and 8 April 2007) in each season were 10.3 and $11.0^{\circ} \mathrm{C}$, respectively. Accumulated light intensity and the range of day length during each season (from seeding date to first inoculation date in 2003-04 and 2006-07) were 1,643 and 1,429 $\mathrm{MJ} / \mathrm{m}^{2}$ and 9.89 to 12.81 and 9.89 to $12.61 \mathrm{~h}$, respectively. The potting soil was prepared by mixing 30 liters of volcanic ash soil obtained from a field of the National Agricultural Research Center for the Kyushu Okinawa Region, 25 liters of vermiculite (Green Sangyo Inc., Saga, Japan), 5 liters of compost (Minori; Eto Sangyo Ltd., Oita, Japan), 4 liters of leaf mold (Green Sangyo Inc.), and $50 \mathrm{ml}$ of calcium magnesium carbonate (Konoha Lime Association, Kumamoto, Japan). Each pot contained $\approx 2$ liters of mixed soil and was fertilized with $2.5 \mathrm{~g}$ of $\mathrm{N}: \mathrm{P}_{2} \mathrm{O}_{5}: \mathrm{K}_{2} \mathrm{O}$ at 8:8:8 (Kumiai-kasei 8; Ryoto Hiryo Co. Ltd., Oita, Japan) and $2 \mathrm{~g}$ of fused magnesium phosphate (National Federation of Agricultural Cooperative Associations, Tokyo, Japan) before seeding, and with $1.4 \mathrm{~g}$ of the $\mathrm{N}: \mathrm{P}_{2} \mathrm{O}_{5}: \mathrm{K}_{2} \mathrm{O}$ at 8:8:8 at the stem elongation stage (Zadoks growth stage [ZGS] 30 to 31) (58). Late tillers of each plant were removed at the heading stage, leaving 6 to 10 similar-stage spikes per pot.

A mixture of two Japanese isolates of $F$. graminearum differing in trichothecene chemotype (i.e., 'H-3' [MAFF Genebank accession no. 101551] of the DON chemotype and 'NIV-2' [MAFF 240548] of the NIV chemotype) was used as the inoculum. In previous in vitro studies, $\mathrm{H}-3$ produced mostly DON and a small amount of NIV, whereas NIV-2 produced mostly NIV and a small amount of DON on a rice medium $(30,31)$. Macroconidia of these

TABLE 1. Treatments performed in the greenhouse experiments ${ }^{\mathrm{a}}$

\begin{tabular}{lcc}
\hline Treatment & Timing of inoculation & Timing of sampling \\
\hline A & - & 10 DAA \\
B & - & 20 DAA \\
C & - & Maturity \\
D & 0 DAA & 10 DAA \\
E & 0 DAA & 20 DAA \\
F & 0 DAA & Maturity \\
G & 10 DAA & 20 DAA \\
H & 10 DAA & Maturity \\
I & 20 DAA & Maturity \\
\hline
\end{tabular}

a Timing: $-=$ noninoculated, DAA $=$ days after anthesis, and Maturity $=38$ to 40 DAA, Zadoks growth stage (ZGS) 92, according to Zadoks et al. (58). isolates were produced by growing the fungus in mung bean liquid medium, as previously described (52). The mung bean liquid medium was prepared as follows: $20 \mathrm{~g}$ of mung bean was boiled in 1 liter of water for $20 \mathrm{~min}$ and $1 \mathrm{~g}$ of yeast extract was added; the broth then was strained and autoclaved.

The obtained macroconidia were suspended in distilled water, the concentration was provisionally adjusted to $2 \times 10^{6}$ spores $/ \mathrm{ml}$, and the macroconidia then stored at $-20^{\circ} \mathrm{C}$ until use. On the days of inoculation, the macroconidia suspensions of the two isolates were thawed and diluted to $5 \times 10^{5}$ spores $/ \mathrm{ml}$ and then mixed in equal volumes. Finally, Tween 20 was added to a final concentration of $0.2 \mathrm{ml} /$ liter.

Treatments differing in the time of inoculation and sampling were conducted as summarized in Table 1. Inoculation was performed at three developmental stages: anthesis ( 0 days after anthesis [DAA]), 10 DAA, and 20 DAA. Sampling was also done at three stages: 10 DAA, 20 DAA, and at maturity (38 to 40 DAA). For each pot, 0 DAA was defined as the day on which all spikes in the pot reached anthesis. Because late tillers were removed at the heading stage, the spikes in each pot flowered almost uniformly. The developmental stages at 10 and 20 DAA corresponded to the early milk (ZGS 73) and late milk (ZGS 77) stages, respectively.

In the experiments, three pots (replicates) per treatment were tested following a completely randomized design, although the inoculation dates were determined by the flowering dates of each cultivar and by the treatments (time of inoculation). The pots for treatments (Trts) D, E, and F (Table 1) and the pots for Trts G and $\mathrm{H}$ (Table 1) of each cultivar were inoculated on the same day in the respective timing. Inoculation was performed between 14:00 and 16:00 on each designated day. Spikes of the potted plants (6 to 10 similar-stage spikes per pot) were spray inoculated with the inoculum at $20 \mathrm{ml} /$ pot using an airbrush (Piece Com PC-WIDE 308; Olympos, Mie, Japan). Thereafter, the inoculated plants were maintained for 6 days under natural light in a glass greenhouse equipped with a sprinkler system. The sprinkler produced a fine mist for 5 min at 15-min intervals between 9:00 and 18:00 and for $5 \mathrm{~min}$ at 2-h intervals between 18:00 and 9:00 to keep the inoculated spikes moist. After inoculation, the pots were rotated within the greenhouse once every 2 days to minimize the effect of location. The plants were then returned to the original greenhouse, where they were grown, with the pots rotated once a week to minimize the effect of location. Accumulated light intensity from the first inoculation date to the last harvest date (3 June in both years) was $897 \mathrm{MJ} / \mathrm{m}^{2}$ in 2004 and $1,109 \mathrm{MJ} / \mathrm{m}^{2}$ in 2007 . Day length during this period was 12.81 to $14.05 \mathrm{~h}$ in 2004 and 12.61 to $14.05 \mathrm{~h}$ in 2007 . The mean temperatures in the glass greenhouse with the sprinkler system and in the original greenhouse during the test period (from the first day of inoculation to the last day of harvest) were 19.3 and $22.0^{\circ} \mathrm{C}$ in 2004 and 20.3 and $21.7^{\circ} \mathrm{C}$ in 2007 , respectively. The daily mean temperature in the glass greenhouse with the sprinkler system during the period used for maintaining the plants in a moist state after inoculation (14 April to 20 May in 2004 and 8 April to 20 May in 2007) ranged between 15.1 and $22.5^{\circ} \mathrm{C}$ in 2004 and 15.7 and $21.7^{\circ} \mathrm{C}$ in 2007 .

FHB disease severity was assessed visually at 10 DAA for Trt D and at 20 DAA for Trts E and G, just before the sampling of spikes at each stage. In addition, FHB severity at 20 DAA was assessed for Trts F, H, and I, in which spikes were sampled at maturity. Only for Emblem in 2007 was FHB severity at 30 DAA also assessed for Trts F, H, and I. In most cases, disease assessment of the spike was not done after 20 DAA because the spikes' natural senescence after 20 DAA masked the appearance of disease symptoms. FHB severity was assessed for each spike on a scale of 0 to $100(0,5,10,20,30,40,50,60,70,80,90$, and 100), according to visual rating of the percentage of discolored area of the spike, as in a previous study (54), and pot averages were calculated. 
Sampling for each treatment was done for all spikes of each pot. The harvested spikes were dried at $70^{\circ} \mathrm{C}$ for $24 \mathrm{~h}$. After drying, the spikes were threshed carefully so that no small or light kernels were lost. During threshing, the light grains from spikes of the same pot were trapped along with all the separated chaff in a net bag that was attached to the opening of the thresher. These grains were separated from the chaff both manually and using sieves. The light grains separated from the chaff were then mixed back in with the heavier grains that were obtained from the plants of the same pot.

The percentage of Fusarium-damaged kernels (FDK) (bleached or discolored and shriveled kernels) and the 1000-grain weight were measured for each grain sample. For the matured samples of 0 - and 10-DAA inoculation (samples of Trts $\mathrm{F}$ and $\mathrm{H}$ ), mean number of grains per spike (GrPS) was calculated using the data of the total number of obtained grains and the number of spikes for each pot. The ratios of GrPS following 0-DAA inoculation to those following 10-DAA inoculation were also calculated for each cultivar in each year.

Field experiments. Seven wheat cultivars ('Chikugoizumi,' Norin 61, 'Shiroganekomugi,' 'Minaminokaori,' 'Nishinokaori,' Sumai 3, and 'Saikai 165') were tested in a field in Koshi, Kumamoto, Japan, in the spring of 2007 and 2008. Chikugoizumi and Shiroganekomugi, as well as Norin 61, are leading Japanese cultivars used to make Japanese noodles. These are soft red wheat types. Minaminokaori and Nishinokaori are Japanese breadmaking cultivars and are hard red wheat types. All these Japanese cultivars are spring wheat genotypes, although they are cultivated as winter wheat in Japan. The levels of FHB resistance are ranked as moderately resistant for Chikugoizumi and Shiroganekomugi, intermediate for Norin 61 and Nishinokaori, and moderately susceptible for Minaminokaori. Saikai 165, which is also a spring wheat genotype, was bred from a cross of Sumai 3 (resistant) and 'Asakazekomugi' (moderately resistant) as an FHB-resistant parent line but its resistance is slightly inferior to that of Sumai 3 (5).

These cultivars were planted in the field in late November 2006 and 2007 in a randomized complete blocks design with three replications. Each plot consisted of 4.5-m long single rows with $80 \mathrm{~cm}$ between rows. The plants were grown in accordance with standard agronomic practices until heading.

Inoculation with $F$. graminearum was performed using colonized maize kernel inoculum $(8,12)$, which generates ascospores over a long period during the testing season in the field. A mixture of four Japanese isolates of $F$. graminearum differing in trichothecene chemotype (i.e., H-3 [MAFF 101551] and 'DON-5' [MAFF 240559] of the DON chemotype and 'NIV-1' [MAFF 240547] and 'NIV-7' [MAFF 240552] of the NIV chemotype) was used. In previous in vitro studies, DON-5 produced only DON, while H-3 produced mostly DON and a small amount of NIV on a rice medium (30,31). NIV-1 and NIV-7 produced mostly NIV and a small amount of DON (31). The virulences of DON-5, NIV-1, and NIV-7 were higher than those of H-3 and NIV-2 (31), which were used in the greenhouse experiments. The colonized maize kernels of each isolate were prepared as described in a previous study (53) and stored at $-20^{\circ} \mathrm{C}$ until use. In mid-March (19 March 2007 and 11 March 2008), the colonized kernels of the four isolates were mixed in equal volumes and distributed in the field by hand at a rate of $\approx 45 \mathrm{~cm}^{3} / \mathrm{m}^{2}$.

Mist irrigation to promote ascospore production and fungal infection was performed in the field using a sprinkler system with DN881A-type sprinkler heads equipped with 1.50-mm-diameter nozzles (Sun Hope Inc., Meguro-ku, Tokyo). The sprinkler system was set up before heading, spacing the sprinkler heads $\approx 2 \mathrm{~m}$ apart, and was activated on 3 April 2007 and 10 April 2008 (at the beginning of head emergence of the earliest cultivar, Chikugoizumi). Water was applied four times daily (at 1:00, 7:00, 13:00, and 19:00 h) for $6 \mathrm{~min}$ at each application at a rate of $\approx 25 \mathrm{~mm} / \mathrm{h}$.
On rainy days, irrigation was not performed. Misting was continued until 25 DAA of the latest cultivar, Sumai 3 (i.e., 21 May 2007 and 25 May 2008), which was the last day of FHB evaluation in each year. The mean temperature in the field during the test period (15 April to 6 June 2007 and 22 April to 10 June 2008) was $17.9^{\circ} \mathrm{C}$ in 2007 and $18.8^{\circ} \mathrm{C}$ in 2008 .

FHB was assessed at 25 DAA (ZGS 77; late milk stage). Fifty arbitrarily selected spikes from each plot were individually assessed on a scale of 0 to 100 as in the greenhouse experiments, and the plot average was calculated. Using these data, FHB incidence $(\%)$ (i.e., the percentage of visually diseased spikes) was also calculated for each plot.

Sampling was done twice, at 20 DAA (late milk stage) and 40 DAA (maturity), for each plot. At each time of sampling, spikes in a row of $1.5 \mathrm{~m}$ (for 20-DAA samples) or $1.0 \mathrm{~m}$ (for mature samples) from the mid-part of each plot were harvested manually and immediately dried at $70^{\circ} \mathrm{C}$ for $24 \mathrm{~h}$. After drying, the spikes were threshed carefully so that no small or light kernels were lost. During threshing, the light grains were trapped along with the separated chaff in a large vat that was placed in front of the opening of the thresher. These grains were separated from the chaff in a manner similar to that of the greenhouse samples and mixed back into the heavier grains from the same plot. For each grain sample, the percentage of FDK $(\%)$ and the 1,000-grain weight were measured.

DON and NIV analyses. For the samples from greenhouse experiments, all grains obtained from each pot were analyzed for DON and NIV content after measuring FDK (\%) and 1,000-grain weight. The amounts of each inoculated sample (per pot) harvested at 10 DAA (= Trt D), 20 DAA (= Trts E and G), and maturity (= Trts F, H, and I) were 1.1 to $2.3,3.7$ to 8.6 , and 8.3 to $16.0 \mathrm{~g}$, respectively. The analyses were performed with an enzyme-linked immunosorbent assay (ELISA) system at the KM Assay Center, Kyowa Medex Co. Ltd. (Nagaizumi-cho, Shizuoka, Japan). Details of the ELISA system and the method of analysis were previously described (57). The quantification limits of the ELISA analyses were $0.1 \mu \mathrm{g} \mathrm{g}^{-1}$ for DON and $0.05 \mu \mathrm{g} \mathrm{g}^{-1}$ for NIV.

For the samples from field experiments, $50 \mathrm{~g}$ (for 20-DAA samples) or $100 \mathrm{~g}$ (for matured grain samples) of grain from each plot were analyzed for DON and NIV. In 2007, the toxin analyses were performed with ELISA in the same way as the greenhouse experiments. In 2008, the toxins were measured by high-performance liquid chromatography (HPLC) according to a standard procedure approved by the Ministry of Health, Labor and Welfare of Japan. The detection limits of DON and NIV in the HPLC analysis were both $0.05 \mu \mathrm{g} \mathrm{g}^{-1}$.

Statistical analyses. In both the greenhouse and the field experiments, to analyze toxin increase from 20 DAA to maturity, the difference in the toxin (DON+NIV) content (micrograms per 100 grains) of samples that differed in the timing of sampling was analyzed with a one-tailed $t$ test for each cultivar in each test year. In the greenhouse experiments, a one-tailed $t$ test was conducted between Trts E and F (following 0-DAA inoculation) and between Trts $\mathrm{G}$ and $\mathrm{H}$ (following 10-DAA inoculation). In the field experiments, a one-tailed $t$ test was conducted between samples harvested at $20 \mathrm{DAA}$ and at maturity. In addition, in the greenhouse experiments, the difference between GrPS following 0-DAA (= Trt F) and that following 10-DAA inoculation (= Trt H) was also analyzed with a one-tailed $t$ test for each cultivar in each test year. All the statistical analyses were performed using Statview (version J5.0; SAS Institute, Cary, NC).

\section{RESULTS}

Progression of disease and toxin accumulation during grain development in the greenhouse experiments. DON and NIV concentrations (micrograms per gram) after 0-DAA inoculation during grain development of the cultivars in 2004 is shown in 
Figure 1. DON and NIV had already accumulated in the grain by 10 days after inoculation $(=\operatorname{Trt} \mathrm{D})$ in all cultivars. The toxin concentration decreased from 10 DAA $(=$ Trt D) to 20 DAA $(=$ Trt E) and increased again after 20 DAA until maturity $(=$ Trt F) in all the cultivars except Nobeokabouzu, which accumulated the lowest amount of these toxins. However, the decrease in toxin concentration (micrograms per gram) between 10 and 20 DAA (= Trts D and E) is not necessarily attributed to an actual toxin decrease in grain (e.g., by degradation, elimination, and so on). This is because grain development of the inoculated plants during 10 to 20 DAA is accompanied by a dramatic increase in weight (Fig. 1), resulting in a decrease in the number of grains per fixed grain weight, which is the denominator of the unit for toxin concentration (micrograms per gram). In order to determine the actual manner in which the toxin increases in developing grain, the toxin content in grain should be shown on a grain-number basis instead of a grain-weight basis.

The data on toxin content of all the inoculated samples $(=$ Trts D to I) are shown on a grain-number basis (micrograms per 100 grains) (Fig. 2). This demonstrated that no apparent decrease in the toxins occurred from 10 to 20 DAA after 0-DAA inoculation (shown between Trts D and E) in all the tested cultivars in both years. In addition, the toxin amount in grain largely increased from 20 DAA to maturity (between Trts E and F, between Trts G and $\mathrm{H}$, and in Trt I), except in a few cases (i.e., Norin 61 inoculated 10 DAA in 2007 and Nobeokabouzu in both years). For example, in Norin 61 in 2004, the toxin amount increased from 20 DAA to maturity by 3.5 -fold after 0-DAA inoculation (between Trts E and F) and by 8.8-fold after 10-DAA inoculation (between Trts $\mathrm{G}$ and $\mathrm{H}$ ). Likewise, in Emblem in 2004, toxin increased from 20 DAA to maturity by 5.4-fold after 0-DAA inoculation and by 5.0-fold after 10-DAA inoculation. Although toxin increase of this period in 2007 was not significant at $P=$ 0.05 for Norin 61 after 0-DAA inoculation (between Trts E and F) and Sumai 3 after 10-DAA inoculation (between Trts $\mathrm{G}$ and $\mathrm{H}$ ), the increases were nearly significant (both $P=0.06$ ) and the numerical increase rates were $\times 2.3$ and $\times 2.5$, respectively.

DON and NIV accumulated in grain following inoculation as late as 20 DAA (= Trt I) in the tested cultivars, with a few exceptions (Figs. 2 and 3). In Nobeokabouzu, only a trace amount of NIV was detected in the grain after 20-DAA inoculation in both years.

Along with the data on toxin content, data on progression of FHB severity and FDK (\%) during grain development are also shown in Figure 2. The FDK of the samples harvested at 10 or
20 DAA included shriveled grain with brownish discoloration, whereas all the FDK of the mature grain samples were bleached and shriveled. The increase in FDK (\%) after inoculation showed a pattern similar to the toxin increase, especially in 2004. FDK (\%) increased from 20 DAA to maturity in most cases (shown between Trts $\mathrm{E}$ and $\mathrm{F}$ and Trts $\mathrm{G}$ and $\mathrm{H}$, and in Trt I), except in Nobeokabouzu.

The noninoculated plants (Trts A to C) did not show any symptoms or toxin accumulation in grain (data not shown).

Influence of infection timing on the disease and final toxin level in the greenhouse experiments. Disease data (i.e., FHB severity at 20 DAA and FDK [\%] at maturity) and toxin concentration (micrograms per gram) in mature grain for the samples inoculated at different stages (i.e., at 0,10 , and 20 DAA) in the greenhouse experiments, all of which were obtained from Trts F, H, and I, are shown in Figure 3. In this figure, toxin concentration data are shown on a grain-weight basis, as is common. This is because all the toxin data are for mature grain and the results do not differ significantly whether they are given on a grain-weight or grain-number basis.

In most cases, FHB severity was highest when plants were inoculated at 0 DAA $(=$ Trt F) and declined with the delay in time of infection. An exception was Nobeokabouzu in 2004, which showed only slight FHB symptoms even after inoculation at 0 DAA. FHB severity with 20-DAA inoculation (= Trt I) was zero in all cases because the severity was evaluated on the day of inoculation.

The decline in FHB severity with delayed infection was not necessarily accompanied by a change in the final FDK (\%) and final toxin concentration, which were assessed on mature grain. In most cases, the final FDK $(\%)$ and final toxin concentration following 10-DAA inoculation $(=\operatorname{Trt} \mathrm{H})$ were not less than those following 0-DAA inoculation (= Trt F). The final FDK $(\%)$ or final toxin concentration following 10-DAA inoculation appeared to be higher than that following 0-DAA inoculation in some cases (i.e., Emblem in both years and Norin 61 and Sumai 3 in 2007). In addition, nonignorable levels of toxin and FDK were detected in the mature samples following 20-DAA inoculation (= Trt I) in some cases, even without clear FHB symptoms on the spikes after inoculation. The final concentration of toxin (DON+NIV) following 20-DAA inoculation (= Trt I) was more than $1.8 \mu \mathrm{g} \mathrm{g}^{-1}$ in five of eight cases. The levels exceed the maximum allowed $\left(1.1 \mu \mathrm{g} \mathrm{g}^{-1}\right)$ for DON content in wheat in Japan. Emblem in 2007 even showed levels of toxin accumulation as high as $17.8 \mu \mathrm{g} \mathrm{g}^{-1}$ following the late infection. In most cases, spikes turned

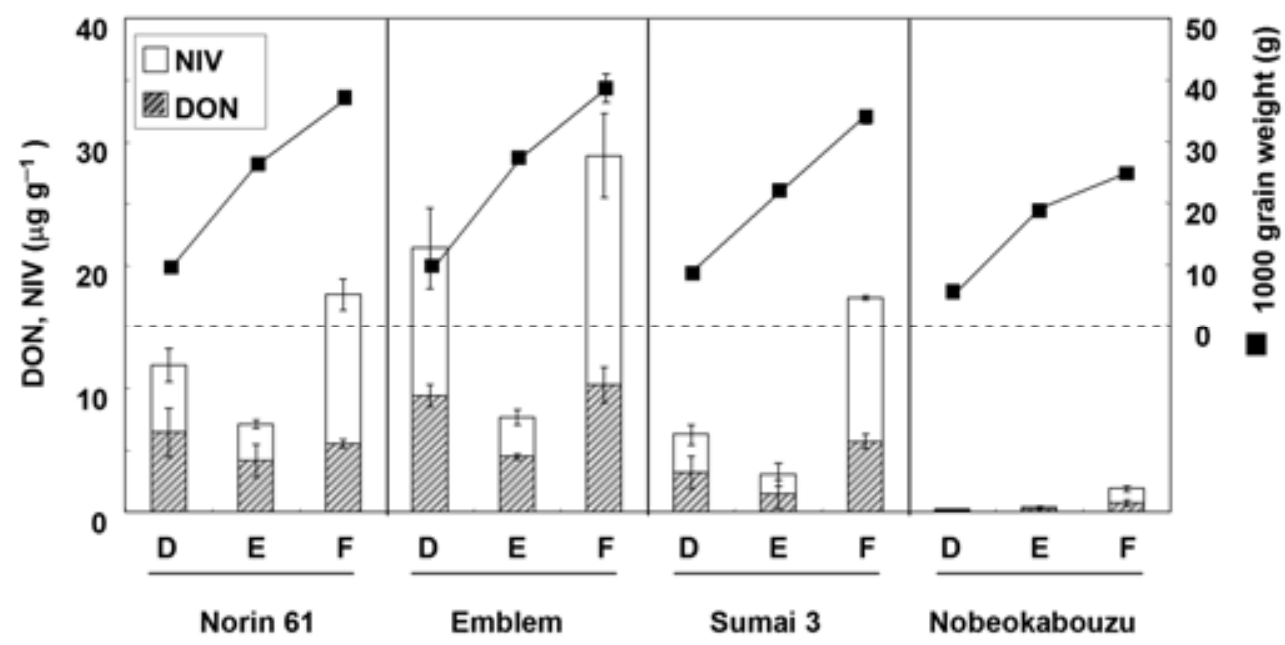

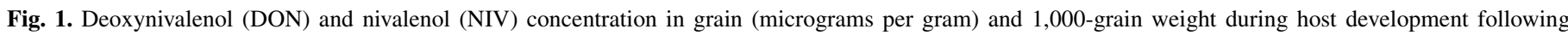

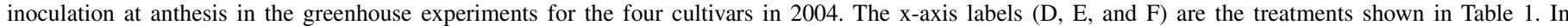

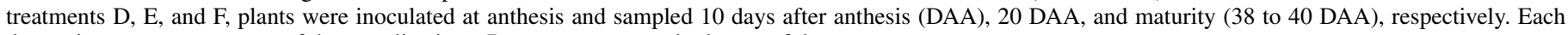
data point represents a mean of three replications. Bars represent standard error of the mean. 
gradually yellow after 20 DAA as natural senescence, and FHB symptoms on the spikes following 20-DAA inoculation (= Trt I) were not clearly observed even after inoculation. Only in Emblem did green color on the spike last as late as 30 DAA, and some clear discoloration was observed on the spike after 20-DAA inoculation. In 2004, discoloration following 20-DAA inoculation
(= Trt I) was only slight but, in 2007, the level of discoloration (FHB severity) at 30 DAA was scored as 47 in Trt I and as 45 and 54 in the treatments with earlier inoculation $(=\operatorname{Trts} \mathrm{F}$ and $\mathrm{H}$, respectively) in Emblem. With regard to resistant cv. Nobeokabouzu, the levels of FDK and toxin accumulation were consistently low.

\section{4}

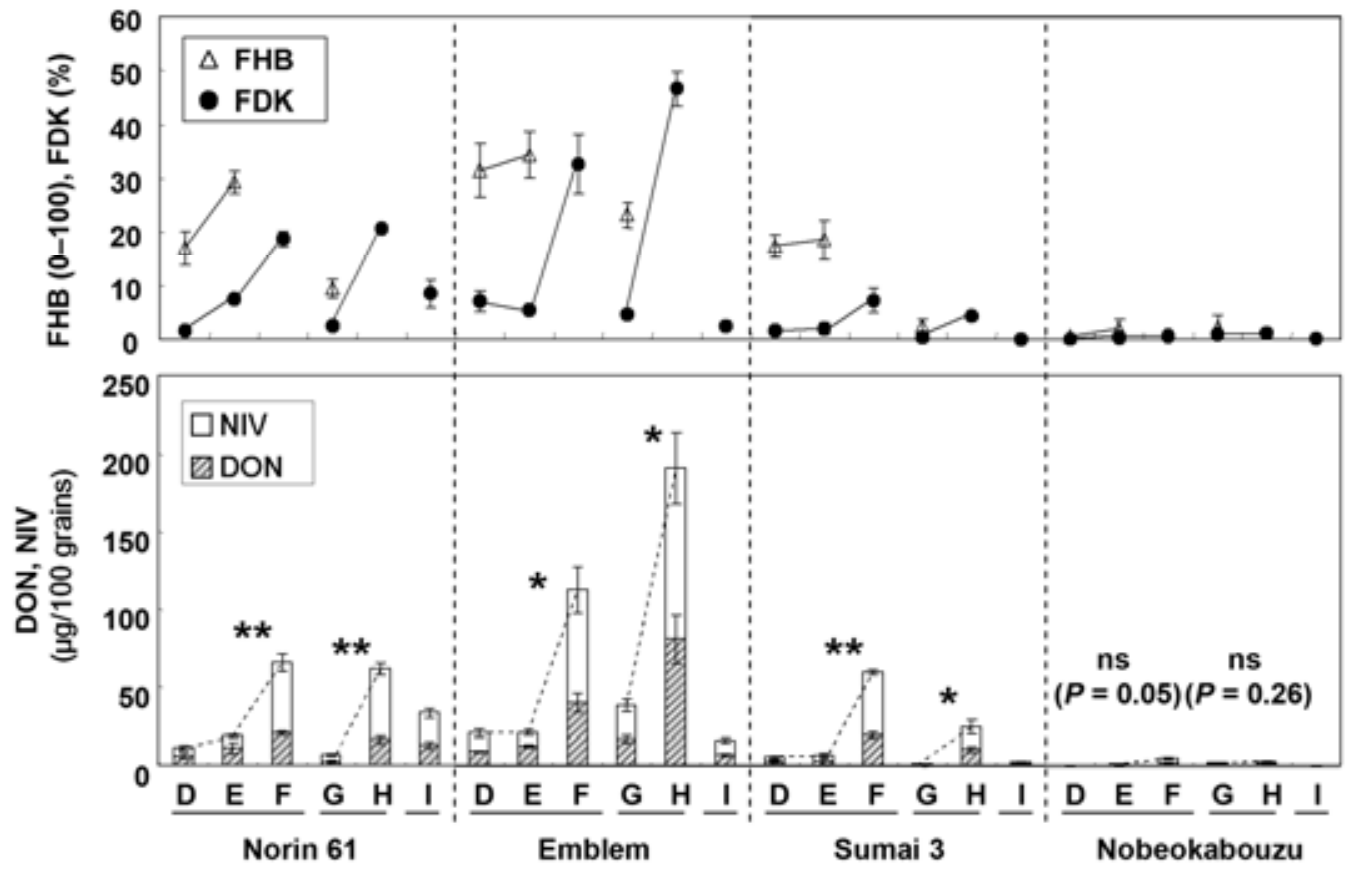

2007

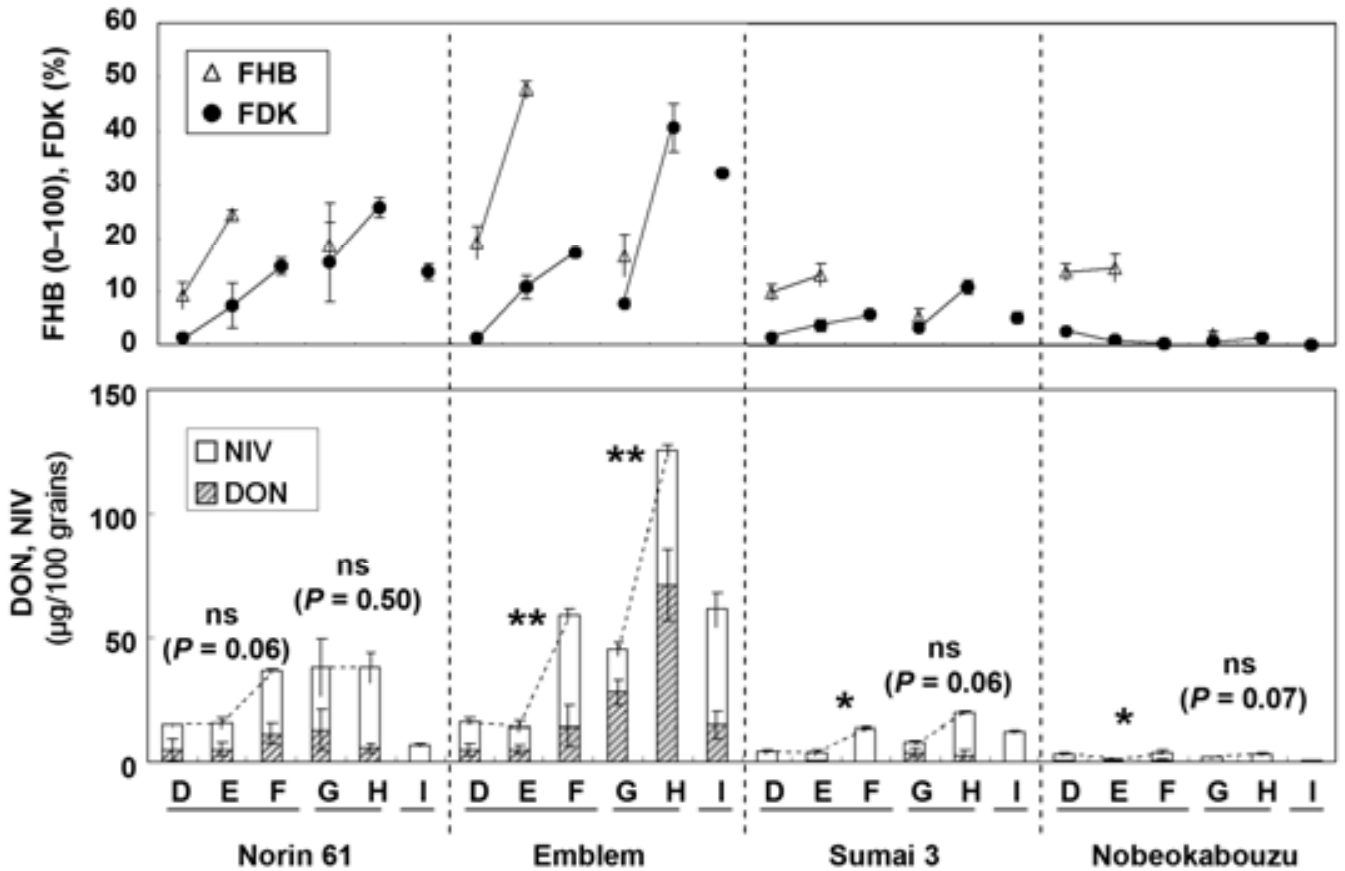

Fig. 2. Deoxynivalenol (DON) and nivalenol (NIV) concentration in grains (micrograms per 100 grains), Fusarium head blight (FHB) severity, and the percentage of Fusarium-damaged kernels (FDK) during host development after different timings of inoculation for the four cultivars in the greenhouse experiments in 2004 and 2007. The $\mathrm{x}$-axis labels (D to I) are the treatments shown in Table 1. In treatments (Trts) D, E, and F, plants were inoculated at anthesis and sampled 10 days after anthesis (DAA), 20 DAA, and maturity (38 to 40 DAA), respectively. In Trts G and H, plants were inoculated at 10 DAA and sampled at 20 DAA and at maturity, respectively. In Trt I, plants were inoculated at 20 DAA and sampled at maturity. FHB severity was assessed on the spikes just before each sampling (i.e., 10 DAA in Trt D and 20 DAA in Trts E and G). Toxin concentration and FDK (\%) for each treatment were assessed on the dry grain samples. Each data point represents a mean of three replications. Bars represent standard error of the mean; * and ** indicate $P<0.05$ and 0.01 , respectively, for differences of toxin $(\mathrm{DON}+\mathrm{NIV})$ concentration in grain (micrograms per 100 grains) between Trts $\mathrm{E}$ and $\mathrm{F}$ and between $\mathrm{Trts} \mathrm{G}$ and $\mathrm{H}$ (one-tailed $t$ test); ns $=$ difference not significant. 
The appearance of FDK was somewhat different among treatments in each cultivar. In the earlier inoculated samples, FDK symptoms were heavier and clearer (i.e., FDKs were somewhat smaller or thinner in size, and the extent of shriveling and discoloration was more severe in the order of $\operatorname{Trts} \mathrm{F} \geq \mathrm{H}>\mathrm{I}$ ) (Fig. 4).

The mean number of mature GrPS following 0- and 10-DAA inoculation $(=$ Trts $\mathrm{F}$ and $\mathrm{H})$ is also shown in Figure 3. In all cases, GrPS following 0-DAA inoculation was, at least numerically, fewer than that following 10-DAA inoculation. The ratios of GrPS following 0-DAA inoculation $(=$ Trt F) to that following 10-DAA inoculation $(=\operatorname{Trt} \mathrm{H})$ for the tested cultivars were 0.74 to 0.91 in 2004 and 0.64 to 0.82 in 2007 (Fig. 3). The difference between GrPS following 0-DAA inoculation and that following 10-DAA inoculation was statistically significant at $P=0.05$ (onetailed $t$ test) for Emblem and Sumai 3 in 2007.
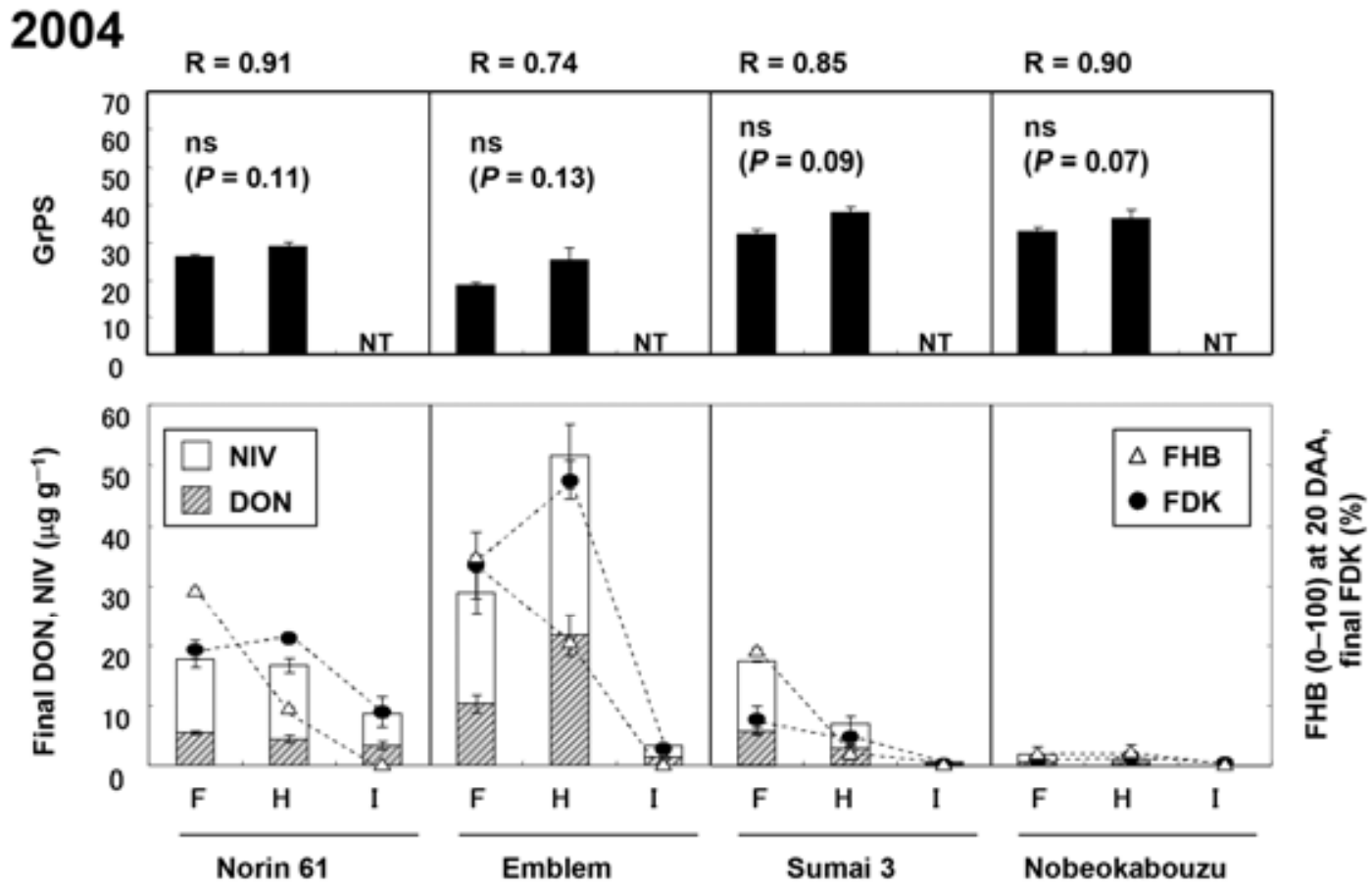

2007
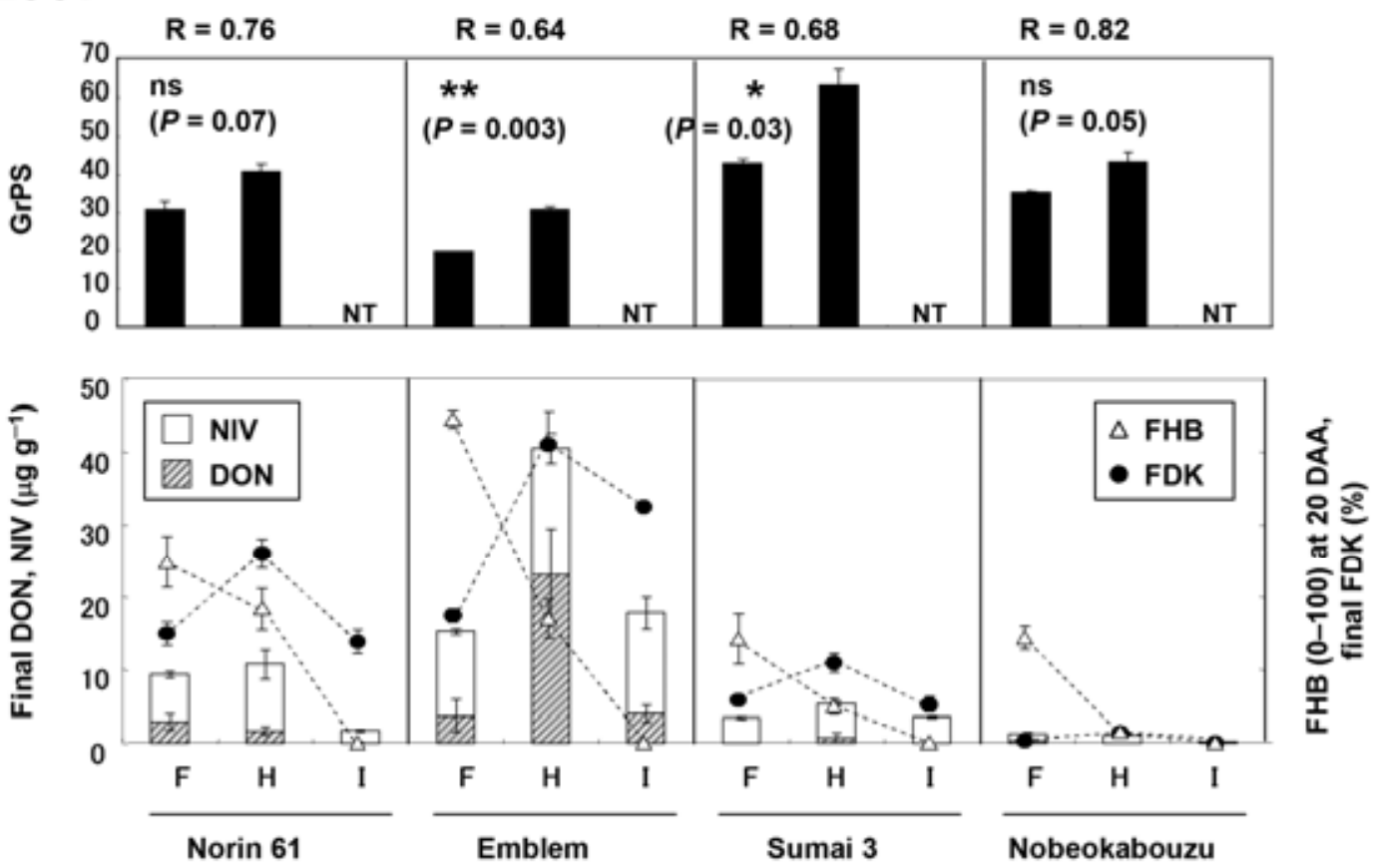

Fig. 3. Fusarium head blight (FHB) severity assessed 20 days after anthesis (DAA), percentage of Fusarium-damaged kernels (FDK) and toxin concentration (micrograms per gram) assessed on mature samples, and number of mature grains per spike (GrPS) following different timings of inoculation for the four cultivars in the greenhouse experiments in 2004 and 2007. Data of treatments (Trts) F, H, and I, listed in Table 1, are shown. In Trts F, H, and I, plants were inoculated at 0, 10, and 20 DAA, respectively, and harvested at maturity (38 to 40 DAA). The x-axis labels represent each treatment. Each data point represents a mean of three replications. Bars represent standard error of the mean; $*$ and $* *$ indicate $P<0.05$ and 0.01 , respectively, for differences of GrPS between the two inoculation times $(=$ Trts $\mathrm{F}$ and $\mathrm{H})$ (one-tailed $t$ test); ns = difference not significant; NT = not tested; $\mathrm{R}=$ ratio of GrPS following 0 DAA inoculation $(=$ Trt F) to that following 10 DAA inoculation $(=\operatorname{Trt} \mathrm{H})$. 
Disease levels and progression of toxin accumulation in the field experiments. The disease levels and final toxin concentration of the tested cultivars in the field experiments are shown in Table 2. For all cultivars, the levels of FDK (\%) and toxin concentration at maturity were higher in 2008 than in 2007.

The progression of toxin accumulation in each cultivar in the field test is shown in Figure 5. In all cases except Sumai 3 in 2008, both DON and NIV were already detected at 20 DAA and the amount largely increased after 20 DAA to maturity (40 DAA). For example, the rates of toxin increases during the period for Chikugoizumi, Norin 61, and Minaminokaori were $\times 4.4, \times 5.9$, and $\times 6.9$ in 2007 and $\times 9.7, \times 8.6$, and $\times 11.5$ in 2008 , respectively. In some cases (i.e., Nishinokaori, Saikai 165, and Sumai 3 in 2007), the increase was not significant at $P=0.05$ but was nearly significant $(P=0.06$ to 0.08$)$, and the numerical increase rates were $\times 2.7, \times 2.4$, and $\times 10.3$, respectively. For Sumai 3 in 2008, DON and NIV were not detected at 20 DAA but, in mature grain, toxin (DON+NIV) at $1.6 \mu \mathrm{g} \mathrm{g}^{-1}$ was detected.

In the field experiments, FDK (\%) at 20 DAA was not measured. This is because the border between FDK and apparently normal kernels was indistinct for the 20-DAA samples, although grains that were slightly discolored (brownish) and shriveled to some extent were found in some 20-DAA samples.

\section{DISCUSSION}

This study examined the manner in which accumulation of NIV as well as DON occurs in wheat grain infected with F. graminearum. We investigated the accumulation of both DON and NIV at the same time because they often co-occur $(36,48,49,55,56)$.
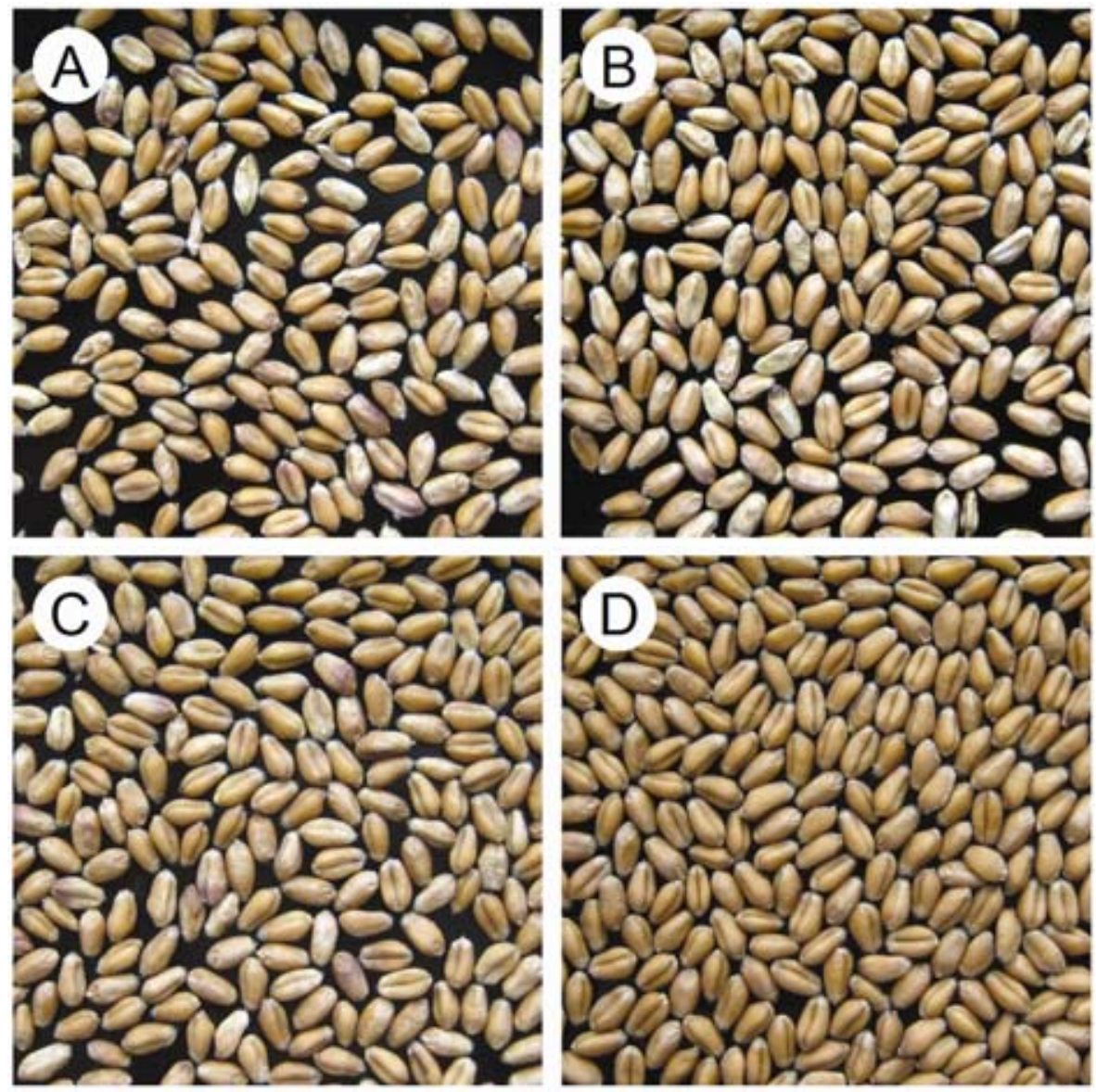

Fig. 4. Appearance of mature grain samples of 'Norin 61' following different treatments in the greenhouse experiments in 2004. A, Inoculated at anthesis (= treatment [Trt] F); B, inoculated 10 days after anthesis (DAA) (= Trt H); C, inoculated 20 DAA (= Trt I); D, noninoculated (= Trt C). Samples A, B, and C contained 18.8, 20.7, and 8.6\% Fusarium-damaged kernels (FDKs) and mycotoxin (deoxynivalenol plus nivalenol) at 17.7, 16.6, and 8.7 $\mu \mathrm{g} \mathrm{g} \mathrm{g}^{-1}$, respectively. No FDK and mycotoxin was detected in the noninoculated sample (sample D).

TABLE 2. Date of anthesis, Fusarium head blight (FHB) severity 25 days after anthesis (DAA), percentage of Fusarium damaged kernels (FDK), and deoxynivalenol (DON) and nivalenol (NIV) concentration (toxin at maturity) in mature samples of the seven cultivars in the field experiments in 2007 and 2008

\begin{tabular}{|c|c|c|c|c|c|c|c|c|c|c|c|c|}
\hline \multirow[b]{3}{*}{ Cultivar } & \multicolumn{6}{|c|}{2007} & \multicolumn{6}{|c|}{2008} \\
\hline & \multirow[b]{2}{*}{ Anthesis } & \multirow[b]{2}{*}{ FHB } & \multirow[b]{2}{*}{$\operatorname{FDK}(\%)$} & \multicolumn{3}{|c|}{ Toxin at maturity $\left(\mu \mathrm{g} \mathrm{g}^{-1}\right)$} & \multirow[b]{2}{*}{ Anthesis } & \multirow[b]{2}{*}{ FHB } & \multirow[b]{2}{*}{ FDK $(\%)$} & \multicolumn{3}{|c|}{ Toxin at maturity $\left(\mu \mathrm{g} \mathrm{g}^{-1}\right)$} \\
\hline & & & & DON & NIV & $\mathrm{DON}+\mathrm{NIV}$ & & & & DON & NIV & $\mathrm{DON}+\mathrm{NIV}$ \\
\hline Norin 61 & 21 April & 16.0 & 4.6 & 0.8 & 1.0 & 1.9 & 28 April & 18.0 & 13.8 & 2.9 & 2.5 & 5.4 \\
\hline Shiroganekomugi & 16 April & 10.8 & 2.2 & 0.1 & 1.0 & 1.1 & 22 April & 9.5 & 6.0 & 1.7 & 1.3 & 3.0 \\
\hline Minaminokaori & 16 April & 13.9 & 5.0 & 1.4 & 0.8 & 2.2 & 24 April & 22.7 & 23.1 & 5.6 & 3.7 & 9.3 \\
\hline Nishinokaori & 16 April & 11.6 & 3.7 & 0.9 & 0.8 & 1.7 & 25 April & 15.4 & 16.3 & 3.1 & 2.3 & 5.5 \\
\hline Mean & $\ldots$ & 10.4 & 3.4 & 0.7 & 0.8 & 1.5 & $\ldots$ & 12.2 & 11.7 & 2.5 & 2.0 & 4.5 \\
\hline
\end{tabular}


We based the unit on grain number (micrograms per 100 grains) instead of grain weight (e.g., micrograms per gram, parts per million, and so on) to show the mode of progression of toxin accumulation in developing grain, considering the influence of grain-weight increase during development.

In the greenhouse experiments, when progression of toxin accumulation was expressed on a grain-weight basis (micrograms per gram), apparent decreases in toxin were observed at 10 to 20 DAA (Fig. 1). However, these were not attributed to a real decrease in toxin quantity but, rather, to a dilution of toxin due to rapid grain development. When the toxin amount was expressed on a grain-number basis (micrograms per 100 grains), no apparent decrease of the toxins was observed during grain development in all experiments (Fig. 2). The results of the greenhouse experiments, in which specific timing (i.e., 0, 10, and 20 DAA) of inoculation was performed on four cultivars, clearly indicated that the amount of DON and NIV in grain largely increased after 20 DAA (i.e., in the latter period of grain development), even by early infection (i.e., 0- and 10-DAA infection). In addition, infection at 20 DAA also caused grain contamination with these toxins.

In the field experiments, inoculation was performed using colonized maize kernel inoculum, which generates ascospores during the testing season (12). Accordingly, the spikes were exposed to infection throughout the period of spike development after heading. In all the cases, the toxin amounts largely increased after 20 DAA to maturity. These results were consistent with results of the greenhouse experiments. In the field experiments, the toxin increase observed after 20 DAA would be the results of both (i) toxin production by the fungus infected at earlier stages and (ii) toxin production by the fungus newly infected after 20 DAA.

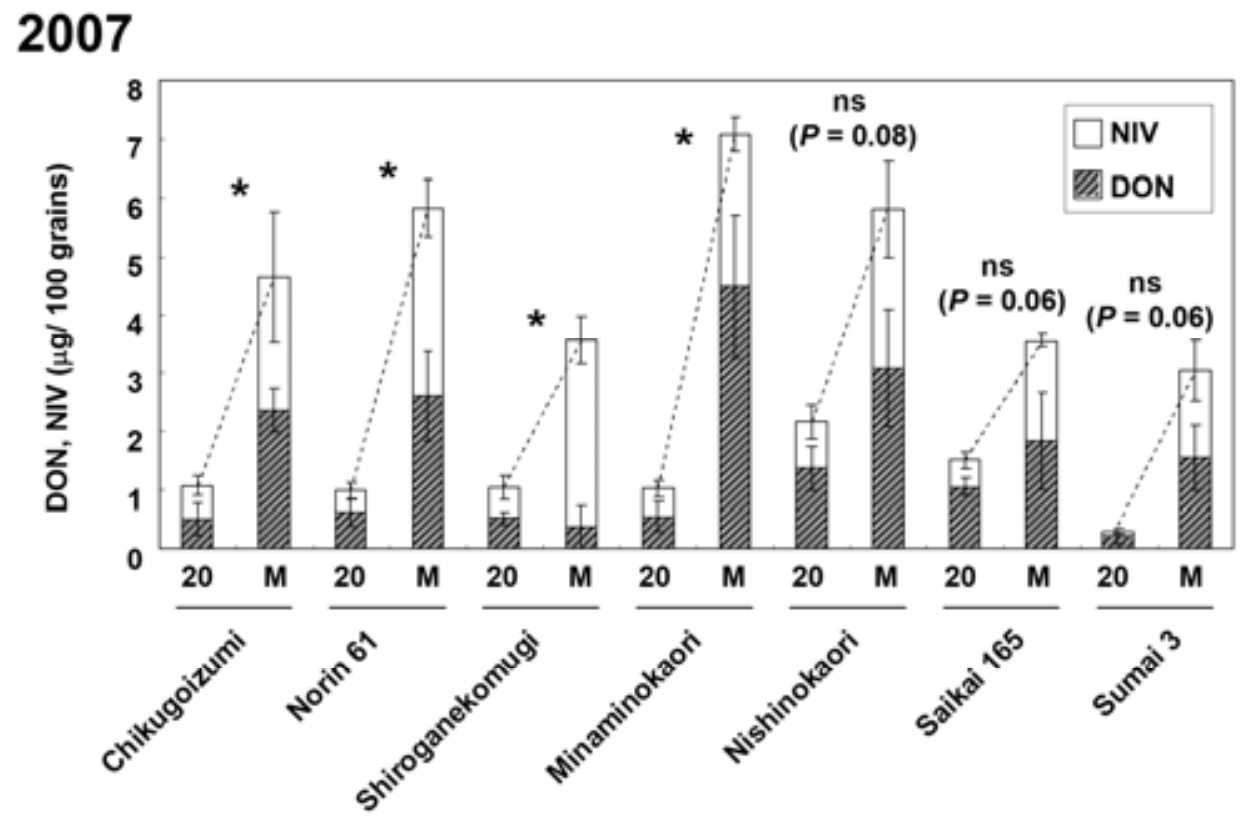

2008

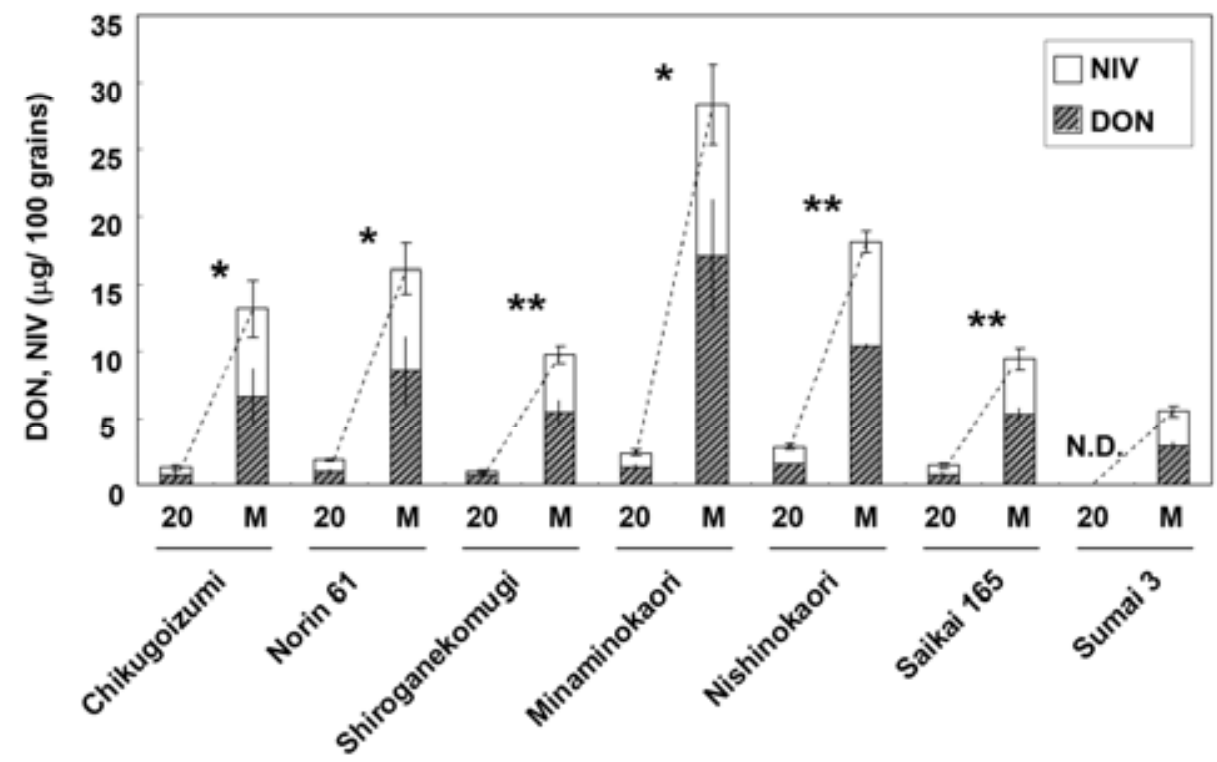

Fig. 5. Deoxynivalenol (DON) and nivalenol (NIV) concentration in grain (micrograms per 100 grains) at two developmental stages for the seven cultivars in the field experiments in 2007 and 2008. Signs below the graph (20 and M) indicate the timing when the samples were harvested, 20 days after anthesis (DAA) and maturity (40 DAA), respectively. Each data point represents a mean of three replications. Bars represent standard error of the mean. N.D. $=$ not detected; $*$ and $* *$ indicate $P<0.05$ and 0.01 , respectively, for differences of toxin (DON+NIV) concentration in grain (micrograms per 100 grains) between the two harvest timings (i.e., 20 DAA and at maturity) (one-tailed $t$ test); ns = difference not significant. 
The levels of FDK (\%) and toxin concentration at maturity were higher in 2008 than in 2007 for all cultivars, although there was no apparent difference in FHB severity between the years. This may be mainly attributed to the difference in the amount of natural precipitation in the late period of the experiments between the years. The total amounts of precipitation from the first day of the FHB assessment to 5 days before the last harvest day were $46.0 \mathrm{~mm}$ in 2007 and $196.0 \mathrm{~mm}$ in 2008 .

Natural infection processes are dependent on environmental conditions in the field, including the pattern of rainfall and provision of inoculum. In addition, the toxin-producing ability of Fusarium isolates varies $(4,27,29,41,45)$, and genotypic diversity within field populations of $F$. graminearum is reported to be high $(14,18,59,60)$. Therefore, the patterns of toxin accumulation may vary in natural fields and between years.

Nevertheless, the results of both of the two distinct experiments in this study, in which inoculations were performed in different ways, indicate that high levels of DON and NIV can be produced after 20 DAA, even by early infection, and, therefore, that the later period of grain development is important to determine final toxin contamination level. This is likely to be common for most wheat cultivars because, in both greenhouse and field experiments, large production of DON and NIV after 20 DAA was consistently observed for most of the tested cultivars, which were quite different in pedigree or origin and in levels of resistance to FHB.

Several studies have investigated the progression of trichothecene mycotoxins, especially for DON, during grain development in wheat $(2,7,26,40)$. However, the results have not been consistent. Unlike the present study, all these previous studies showed mycotoxin concentration in grain on a grain-weight basis and, in some studies, F. culmorum was used as inoculum of FHB.

Matthäus et al. (26) reported that DON concentration in grain after spray inoculation with $F$. culmorum increased from 9 days after inoculation (DAI) (early milk) to 44 DAI (full ripeness) without any apparent decline in their field experiment. In the study by Argyris et al. (2), where wheat cultivars were inoculated with $F$. graminearum by distributing infected corn seed in a mistirrigated field, DON was present at significant levels as early as 10 DAA, and the concentration remained within a narrow range throughout grain development. These reports $(2,26)$ seem consistent with our results, which showed that toxin amount (on a grainnumber basis) largely increased in the later period of grain development.

On the other hand, some researchers have found a decline in mycotoxin concentration in developing grain. Scott et al. (40) observed a dramatic decline in DON concentration in developing wheat grain from naturally infected fields in Ontario in 1983. In the 1-year study by Busko et al. (7), in which wheat cultivars were inoculated with $F$. culmorum, maximum concentration of DON or NIV in grain was obtained at 21 DAI, and the concentration largely declined after that. These data $(7,40)$ appear to indicate an actual decrease in mycotoxin amount in developing grain, which is inconsistent with our results and those of Argyris et al. (2) and Matthäus et al. (26). The decline of toxin concentration observed in the former studies $(7,40)$ may be partly due to an increase in grain dry weight and, possibly, loss of heavily damaged grain during the period. However, these possible factors do not appear to fully explain the considerable decline of toxin concentration observed in their studies. Scott et al. (40) noted that the observed DON decline in their study might be a result of a reaction with plant components or metabolism by host plant enzymes. They also noted that degradation by bacteria or other microorganisms could not be ruled out.

Further research is needed to define the conditions or factors related to production and possible reduction of mycotoxins in grain during development. In such research, to understand the actual increase or decrease of mycotoxin in grain during develop- ment, we consider that the data of toxin amount need to be shown on a grain-number basis. In addition, attention should be paid to the treatments after harvesting of the samples. The method of drying after harvest, especially for immature samples, and possible loss of small or light grain during threshing would affect the data on mycotoxin content. In the present study, in all the experiments, the harvested samples were immediately dried at $70^{\circ} \mathrm{C}$ for $24 \mathrm{~h}$ to prevent further toxin production by the fungus, and attention was paid to avoid the loss of small or light grains during threshing. In most previous studies $(7,26,40)$, details of the treatment after harvest are not fully described.

With regard to the influence of infection timing on mycotoxin accumulation in grain, some studies have previously reported that DON can be accumulated in wheat grain by infection as late as the dough stage $(11,15,21)$. In this study, we investigated the influence of infection timing on the disease and final levels of DON and NIV in the greenhouse experiments. Although we tested the effect of infection only as late as 20 DAA (late milk stage), the results for DON and NIV accumulation were in agreement with the previous studies: these toxins accumulated in grain after infection as late as 20 DAA.

The levels of toxin accumulation following 20-DAA inoculation were not stable between years. For example, in Emblem, the final concentration of DON+NIV following 20-DAA inoculation was much lower than that following 10-DAA inoculation but, in 2007, the final concentration of DON+NIV following 20-DAA inoculation was almost half of that following 10-DAA inoculation. Such differences may have been due to minor differences in the developmental stage of the host plant at the time of 20-DAA inoculation or in testing conditions between years, although the temperature during the 6 days after 20-DAA inoculation, in which the spikes were kept moist, did not differ much between the years. For a more quantitative evaluation of the response of each cultivar to late infection, as well as early stages of infection, further detailed studies are needed. Nevertheless, the present study clearly indicates that some level of DON and NIV could accumulate after infection as late as $20 \mathrm{DAA}$, at least in some cultivars.

The pattern of the final FDK (\%) and final toxin concentration did not necessarily correspond to the pattern of FHB severity assessed at 20 DAA, which sharply declined with a delay in infection timing (Fig. 3). The final FDK (\%) and final toxin concentration following 10-DAA inoculation were not less than those following 0-DAA inoculation in most cases. Furthermore, in some cases, the final FDK (\%) or final toxin concentration following 10-DAA inoculation was higher than that following 0 -DAA inoculation. One cause may be that part of the grains that are heavily damaged by 0-DAA inoculation were too shriveled and were lost before maturity, leading to a toxin reduction in the sample. The consistent results that GrPS following 0-DAA inoculation was, at least numerically, fewer than that following 10-DAA inoculation (Fig. 3) support this suggestion. It is reasonable that susceptible cv. Emblem, which consistently showed the lowest ratios of GrPS following 0-DAA inoculation to that following 10-DAA inoculation (0.74 in 2004 and 0.64 in 2007) among the tested cultivars, also had much lower accumulation of DON+NIV by 0-DAA inoculation than by 10-DAA inoculation in both years. Del Ponte et al. (11) reported similar results in their greenhouse experiments using a highly susceptible cultivar, 'Norm' (i.e., DON accumulation in plants inoculated at flowering stage [ZGS 65] was lower than those inoculated past flowering to late milk stages [ZGS 71 to 77]).

In the present study, some levels of FDK and toxin accumulation were caused by 20-DAA inoculation. However, in most cases (except Emblem in 2007), this timing of inoculation did not cause clear FHB symptoms on the spikes even after inoculation. This would mostly be because of the natural senescence of spikes after 20 DAA, when gradual natural yellowing of the spikes 
masked the appearance of disease symptoms. However, because symptoms on grain and toxin accumulation were detected, the fungus would have actually infected spikes with 20-DAA inoculation, even if there were no clear symptoms on spikes. Further histological studies are needed to elucidate how such late infection occurs on spikes and on grain.

The results presented here would mean that, even if the FHB severity assessed in a natural field is absent or very low, DON and NIV can accumulate in the mature grain by late infection, and the accumulated levels of these toxins may not necessarily be low. Previous studies $(11,21)$ also reported DON accumulation in grain without visible symptoms on spikes following Fusarium infection at the late stage.

It has been reported that DON concentration in harvested grain does not consistently correlate with disease severity $(25,27$, 34,43 ), although a meta-analysis using data from 163 studies (34) showed that, overall, significant positive relationships were observed between disease severity and toxin concentration in harvested grain. This can be attributed to differences in the wheat cultivars $(25,27,43)$, Fusarium isolates $(28,34)$, weather conditions $(27,34)$, and other factors (34). Our present results suggest that the difference in infection timing, although it is related to weather conditions, contributes to the inconsistency in the relationship between disease and final toxin content, at least in some cultivars. In years in which considerable level of late infection occurred, DON and NIV may be accumulated more than expected from FHB severity observed in the field.

Strategies for controlling FHB and toxin contamination have been developed, mostly focusing on infection during the flowering stage of the host. However, our present study indicates the importance of the late stages of grain development for toxin contamination in addition to the early stage, showing that (i) the amount of DON and NIV largely increase in grain after 20 DAA (late milk stage), even with infection at earlier stages, and (ii) infection at late stages, at least as late as 20 DAA in this study, can cause nonignorable levels of contamination with these toxins in grain, even without clear FHB symptoms. Thus, control strategies should be established covering the late stage as well as the time around the flowering stage to effectively reduce the risk of DON and NIV contamination.

Application of fungicides or other control agents at the late stage may be an effective measure for reducing the final level of toxin accumulation. Agents used for such "late control" are expected to possess effects not only to prevent primary infection at late stages but also to prevent toxin production in late stages by previously infected fungus. Studies to test the effectiveness of such late control for reducing toxin contamination are needed. The use of resistant cultivars with a lower risk of toxin contamination throughout the developmental period would also be important. To properly assess the total risk of toxin contamination in wheat cultivars, inoculation tests that cover the late as well as the early stages would be required. Among the tested cultivars in this study, Nobeokabouzu, which is an FHB-resistant cultivar widely used in FHB-resistance breeding, as well as Sumai 3 $(38,42)$, showed the highest resistance to mycotoxin accumulation for all the timings of inoculation (Fig. 3). Nobeokabouzu seems to possess consistent resistance to mycotoxin accumulation as well as to FHB during grain development. Such a cultivar may be a useful resistance source, especially for introducing resistance to mycotoxin accumulation during all grain development stages.

\section{ACKNOWLEDGMENTS}

This study was partly funded by the Ministry of Agriculture, Forestry, and Fisheries of Japan, as part of the Integrated Research Program for Functionality and Safety of Food toward an Establishment of Healthy Diets. We thank N. Kawada and the members of the wheat and barley breeding unit of the National Agricultural Research Center for the
Kyushu Okinawa Region for supplying the seed of some cultivars used in this study and the staff of the research team for FHB control at KONARC for technical assistance.

\section{LITERATURE CITED}

1. Anonymous. 2002. Discussion paper on deoxynivalenol. [Agenda Item 16(g), CX/FAC 02/29.] Codex Committee on Food Additives and Contaminants, 34th Session, Rotterdam. Codex Alimentarius Commission FAO/WHO, Rome.

2. Argyris, J., Sanford, D. V., and TeKrony, D. 2003. Fusarium graminearum infection during wheat seed development and its effect on seed quality. Crop Sci. 43:1782-1788.

3. Atanasoff, D. 1920. Fusarium-blight (scab) of wheat and other cereals. J. Agric. Res. 20:1-40.

4. Atanassov, Z., Nakamura, C., Mori, N., Kaneda, C., Kato, H., Jin, Y. Z., Yoshizawa, T., and Murai, K. 1994. Mycotoxin production and pathogenicity of Fusarium species and wheat resistance to Fusarium head blight. Can. J. Bot. 72:161-167.

5. Ban, T., and Suenaga, K. 2000. Genetic analysis of resistance to Fusarium head blight caused by Fusarium graminearum in Chinese wheat cultivar Sumai 3 and the Japanese cultivar Saikai 165. Euphytica 113:87-99.

6. Buerstmayr, H., Ban, T., and Anderson, J. A. 2009. QTL mapping and marker-assisted selection for Fusarium head blight resistance in wheat: a review. Plant Breed. 128:1-26.

7. Busko, M., Perkowski, J., Wiwart, M., Góral, T., Suchowilska, E., Stuper, K., and Matysiak, A.. 2008. Kinetics of fungal metabolites formation after inoculation of wheat spikes with F. culmorum. Cereal Res. Commun. 36 (Suppl. B):443-449.

8. Campbell, K. A. G., and Lipps, P. E. 1998. Allocation of resources: sources of variation in Fusarium head blight screening nurseries. Phytopathology 88:1078-1086.

9. Cowger, C., Patton-Özkurt, J., Brown-Guedira, G., and Perugini, L. 2009. Post-anthesis moisture increased Fusarium head blight and deoxynivalenol levels in North Carolina winter wheat. Phytopathology 99:320-327.

10. Culler, M. D., Miller-Garvin, J. E., and Dill-Macky, R. 2007. Effect of extended irrigation and host resistance on deoxynivalenol accumulation in Fusarium-infected wheat. Plant Dis. 91:1464-1472.

11. Del Ponte, E. M., Fernandes, J. M. C., and Bergstrom, G. C. 2007. Influence of growth stage on Fusarium head blight and deoxynivalenol production in wheat. J. Phytopathol. 155:577-581.

12. Dill-Macky, R. 2003. Inoculation methods and evaluation of Fusarium head blight resistance in wheat. Pages 184-210 in: Fusarium Head Blight of Wheat and Barley. K. J. Leonard and W. R. Bushnell, eds. The American Phytopathological Society, St. Paul, MN.

13. Fernando, W. G. D., Paulitz, T. C., Seaman, W. L., and Martin, R. A. 1997. Fusarium head blight susceptibility of wheat inoculated at different growth stages. (Abstr.) Phytopathology 87:S30.

14. Gale, L. R., Chen, L. F., Hernick, C. A., Takamura, K., and Kistler, H. C. 2002. Population analysis of Fusarium graminearum from wheat fields in eastern China. Phytopathology 92:1315-1322.

15. Hart, L. P., Pestka, J. J., and Liu, M. T. 1984. Effect of kernel development and wet periods on deoxynivalenol in wheat infected with Gibberella zeae. Phytopathology 74:1415-1418.

16. Ichinoe, M., Hagiwara, H., and Kurata, H. 1984. Distribution of trichothecene-producing fungi in barley and wheat fields in Japan. Pages 190-198 in: Toxigenic Fungi: Their Toxins and Health Hazard. H. Kurata and Y. Ueno, eds. Elsevier, Kodansha Ltd., Tokyo, Amsterdam, New York.

17. Ichinoe, M., Kurata, H., Sugiura, Y., and Ueno, Y. 1983. Chemotaxonomy of Gibberella zeae with special reference to production of trichothecenes and zearalenone. Appl. Environ. Microbiol. 46:1364-1369.

18. Karugia, G. W., Suga, H., Gale, L. R., Nakajima, T., Tomimura, K., and Hyakumachi, M. 2009. Population structure of the Fusarium graminearum species complex from a single Japanese wheat field sampled in two consecutive years. Plant Dis. 93:170-174.

19. Kim, H. S., Lee, T., Dawlatana, M., Yun, S. H., and Lee, Y. W. 2003. Polymorphism of trichothecene biosynthesis genes in deoxynivalenoland nivalenol-producing Fusarium graminearum isolates. Mycol. Res. 107:190-197.

20. Koizumi, S., Kato, H., Yoshino, R., Komada, H., Ichinoe, M., Umehara, Y., and Hayashi, N. 1997. Pathogenicity and ecology of Fusarium species associated with wheat and barley scab in Japan. Bull. Inst. Compr. Agric. Sci. Kinki Univ. 5:31-45.

21. Lacey, J., Bateman, G. L., and Mirocha, C. J. 1999. Effects of infection time and moisture on development of ear blight and deoxynivalenol production by Fusarium spp. in wheat. Ann. Appl. Biol. 134:277-283.

22. Lee, T., Han, Y. K., Kim, K. H., Yun, S. H., and Lee, Y. W. 2002. Tri 13 and Tri 7 determine deoxynivalenol- and nivalenol-producing chemotypes of Gibberella zeae. Appl. Environ. Microbiol. 68:2148-2154. 
23. Lemmens, M., Buerstmayr, H., Krska, R., Schuhmacher, R., Grausgruber, H., and Ruckenbauer, P. 2004. The effect of inoculation treatment and long-term application of moisture on Fusarium head blight symptoms and deoxynivalenol contamination in wheat grains. Eur. J. Plant Pathol. 110:299-308.

24. Liddell, C. M. 2003. Systematics of Fusarium species and allies associated with Fusarium head blight. Pages 35-43 in: Fusarium Head Blight of Wheat and Barley. K. J. Leonard and W. R. Bushnell, eds. The American Phytopathological Society, St. Paul, MN.

25. Liu, W., Langseth, W., Skinnes, H., Elen, O. N., and Sundheim, L. 1997. Comparison of visual head blight ratings, seed infection levels, and deoxynivalenol production for assessment of resistance in cereals inoculated with Fusarium culmorum. Eur. J. Plant Pathol. 103:589-595.

26. Matthäus, K., Dänicke, S., Vahjen, W., Simon, O., Wang, J., Valenta, H., Meyer, K., Strumpf, A., Ziesenib, H., and Flachowsky, G. 2004. Progression of mycotoxin and nutrient concentrations in wheat after inoculation with Fusarium culmorum. Arch. Anim. Nutr. 58:19-35.

27. Mesterházy, Á. 2002. Role of deoxynivalenol in aggressiveness of Fusarium graminearum and F. culmorum and in resistance to Fusarium head blight. Eur. J. Plant Pathol. 108:675-684.

28. Mesterházy, Á., Bartók, T., Mirocha, C. G., and Komoróczy, R. 1999. Nature of wheat resistance to Fusarium head blight and the role of deoxynivalenol for breeding. Plant Breed. 118:97-110.

29. Mirocha, C. J., Abbas, H. K., Windels, C. E., and Xie, W. 1989. Variation in deoxynivalenol, 15-deoxynivalenol, 3-acetyldeoxynivalenol and zearalenone production by Fusarium graminearum isolates. Appl. Environ. Microbiol. 55:1315-1316.

30. Miyasaka, A., Koizumi, S., Imazeki, M., Yasuda, N., Imazaki, I., and Kawakami, A. 2008. Pathogens associated with Fusarium head blight of wheat and barley in the eastern part of Japan and their mycotoxin productivity. Bull. Natl. Agric. Res. Cent. 11:191-199. (In Japanese with English summary)

31. Nakajima, T., and Yoshida, M. 2007. Mycotoxin productivity and virulence of Fusarium graminearum species complex causing Fusarium head blight on wheat and barley in the western part of Japan. Jpn. J. Phytopathol. 73:106-111. (In Japanese with English abstract)

32. Nishikado, Y. 1958. Studies on the wheat scab, caused by Gibberella zeae (Schw.) Petch, and its control. Nougyou Kairyou Gijutsu Shiryou 97:1162. (In Japanese with English summary)

33. Parry, D. W., Jenkinson, P., and McLeod, L. 1995. Fusarium ear blight (scab) in small-grain cereals: a review. Plant Pathol. 44:207-238.

34. Paul, P. A., Lipps, P. E., and Madden, L.V. (2005) Relationship between visual estimates of Fusarium head blight intensity and deoxynivalenol accumulation in harvested wheat grain: a meta-analysis. Phytopathology 95:1225-1236.

35. Pirgozilev, S. R., Edwards, S. G., Hare, M. C., and Jenkinson, P. 2003. Strategies for the control of Fusarium head blight in cereals. Eur. J. Plant Pathol. 109:731-742.

36. Placinta, C. M., D’Mello, J. B. F., and Macdonald, A. M. C. 1999. A review of world contamination of cereal grains and animal feeds with Fusarium mycotoxins. Anim. Feed Sci. Technol. 78:21-37.

37. Pugh, G. W., Johann, H., and Dickson, J. G. 1933. Factors affecting infection of wheat heads by Gibberella saubinetii. J. Agric. Res. 46:771-797.

38. Rudd, J. C., Horsley, R. D., McKendry, A. L., and Elias, E. M. 2001. Host plant resistance genes for Fusarium head blight: sources, mechanisms, and utility in conventional breeding systems. Crop Sci. 41:620-627.

39. Ryu, J., Ohtsubo, K., Izumiyama, N., Nakamura, K., Tanaka, T., Yamamura, H., and Ueno, Y. 1988. The acute and chronic toxicities of nivalenol in mice. Fundam. Appl. Toxicol. 11:38-47.

40. Scott, P. M., Nelson, K., Kanhere, S. R., Karpinski, K. F., Hayward, S., Neish, G. A., and Teich, A. H. 1984. Decline in deoxynivalenol (vomitoxin) concentrations in 1983 Ontario winter wheat before harvest. Appl. Environ. Microbiol. 48:884-886.

41. Seo, J. A., Kim, J. C., Lee, D. H., and Lee, Y. W. 1996. Variation in 8-keto-trichothecenes and zearalenone production by Fusarium graminearum isolates from corn and barley in Korea. Mycopathologia 134:31-37.

42. Shi, J. R., Xu, D. H., Yang, H. Y., Lu, Q. X., and Ban, T. 2008. DNA marker analysis for pyramided of Fusarium head blight (FHB) resistance QTLs from different germplasm. Genetica 133:77-84.

43. Snijders, C. H. A., and Perkowski, J. 1990. Effects of head blight caused by Fusarium culmorum on toxin content and weight of wheat kernels. Phytopathology 80:566-570.

44. Suga, H., Karugia, G. W., Ward, T., Gale, L. R., Tomimura, K., Nakajima, T., Miyasaka, A., Koizumi, S., Kageyama, K., and Hyakumachi, M. 2008. Molecular characterization of the Fusarium graminearum species complex in Japan. Phytopathology 98:159-166.

45. Sugiura, Y., Watanabe, Y., Tanaka, T., Yamamoto, S., and Ueno, Y. 1990. Occurrence of Gibberella zeae strains that produce both nivalenol and deoxynivalenol. Appl. Environ. Microbiol. 56:3047-3051.

46. Sutton, J. C. 1982. Epidemiology of wheat head blight and maize ear rot caused by Fusarium graminearum. Can. J. Plant Pathol. 4:195-209.

47. Takahashi, M., Shibutani, M., Sugita-Konishi, Y., Aihara, M., Inoue, K., Woo, G., Fujimoto, H., and Hirose, M. 2008. A 90-day subchronic toxicity study of nivalenol, a trichothecene mycotoxin, in F344 rats. Food Chem. Toxicol. 46:125-135.

48. Tanaka, T., Hasegawa, A., Yamamoto, S., Lee, U. S., Sugiura, Y., and Ueno, Y. 1988. Worldwide contamination of cereals by the Fusarium mycotoxins nivalenol, deoxynivalenol, and zearalenone. 1. Survey of 19 countries. J. Agric. Food Chem. 36:979-983.

49. Tanaka, T., and Sugiura, Y. 2003. Levels and pattern of contamination with trichothecenes in cereal grains and their risk assessment. Mycotoxins 53:119-121.

50. Ueno, Y., Aikawa, Y., Okumura, H., Sugiura, Y., Nakamura, K., Masuma, R., Tanaka, T., Young, C. J., and Savard, M. E. 1997. Trichothecenes produced by Fusarium species Fn 2B. Mycotoxins 45:25-31.

51. Ward, T. J., Bielawski, J. P., Kistler, H. C., Sullivan, E., and O’Donnell, K. 2002. Ancestral polymorphism and adaptive evolution in the trichothecene mycotoxin gene cluster of phytopathogenic Fusarium. Proc. Natl. Acad. Sci. USA 99:9278-9283.

52. Yoshida, M., Kawada, N., and Nakajima, T. 2007. Effect of infection timing on Fusarium head blight and mycotoxin accumulation in open- and closed-flowering barley. Phytopathology 97:1054-1062.

53. Yoshida, M., Nakajima, T., Arai, M., Suzuki, F., and Tomimura, K. 2008. Effect of the timing of fungicide application on Fusarium head blight and mycotoxin accumulation in closed-flowering barley. Plant Dis. 92:11641170.

54. Yoshida, M., Nakajima, T., and Tonooka, T. 2008. Effect of nitrogen application at anthesis on Fusarium head blight and mycotoxin accumulation in breadmaking wheat in the western part of Japan. J. Gen. Plant Pathol. 74:355-363.

55. Yoshizawa, T. 1997. Geographic difference in trichothecene occurrence in Japanese wheat and barley. Bull. Inst. Compr. Agric. Sci. Kinki Univ. 5:23-30.

56. Yoshizawa, T., and Jin, T. Z. 1998. Trichothecene occurrence in Japanese wheat and barley-its characteristics. Mycotoxins 47:15-18.

57. Yoshizawa, T., Kohno, H., Ikeda, K., Shinoda, T., Yokohama, H., Morita, K., Kusada, O., and Kobayashi, Y. 2004. A practical method for measuring deoxynivalenol, nivalenol, and T-2 + HT-2 toxin in foods by an enzyme-linked immunosorbent assay using monoclonal antibodies. Biosci. Biotechnol. Biochem. 68:2076-2085.

58. Zadoks, J. C., Chang, T. T., and Konzak, C. F. 1974. A decimal code for the growth stages of cereals. Weed Res. 14:415-421.

59. Zeller, K. A., Bowden, R. L., and Leslie, J. F. 2003. Diversity of epidemic populations of Gibberella zeae from small quadrats in Kansas and North Dakota. Phytopathology 93:874-880.

60. Zeller, K. A., Bowden, R. L., and Leslie, J. F. 2004. Population differentiation and recombination in wheat scab populations of Gibberella zeae from the United States. Mol. Ecol. 13:563-571. 